\title{
The Method of Research Subject Chosen and Clinical Orientation in the Secondary Development of Famous and Excellent Chinese Patent Medicines
}

\section{Shang Hongcai*}

Tianjin University of Traditional Chinese Medicine, Tianjin, China

\begin{abstract}
Secondary development of famous and excellent Chinese patent medicines is one of the principal pathways for significant new drug creation. A well-chosen topic for in-depth study and a more precise definition of the drug's medicinal uses and indications play a decisive role to successful drug redevelopment. The author puts forward a four-step method of "grasping the baseline information of target diseases and related Chinese patent medicines, choosing an initial research topic and orientating the medicine in question to a specific clinical use, analyzing the scientificity and risk of the study and making a strategic decision for the research", followed by explanations and illustrations in a specific case study.
\end{abstract}

Keywords: Chinese patent medicines; Secondary development; Research subject choosing; Clinical orientation

\section{Introduction}

Recent years have witnessed a shift of pattern in drug development from new drug creation to the secondary development of time-enduring quality drugs, namely, to obtain new drugs with upgraded efficacy by optimizing the formula of a selection of famous and excellent Chinese patent medicines [1].

In early 2008, "significant new drug creation" was listed among one of the 16 national major science and technology special projects in China's mid-and long-term development plan. The secondary development of Chinese patent medicine was especially highlighted in the "traditional Chinese medicine innovative development and planning framework (2006-2020)" co-issued by 16 national ministries and commissions, which encouraged "in-depth systematic study and (secondary) development of traditional Chinese medicine with proven effects". As an important way of creating new drugs, the goal of secondary development of famous and excellent Chinese patent medicine is to restudy post-marketing drugs, enrich it with more scientific content, and optimize its quality for the benefits of the patients.

The work of secondary development needs to be done on the basis of previous experiments and clinical evaluations, as well as with the aid of advanced technologies in chemistry pharmacy, practical method of quality control and state-of-the-art equipments. However, it has been observed that in practice each part of drug development acts on its own due to lack of overall management and previous literature on a chosen subject was not well summarized. Moreover, there are some problems about choosing an appropriate patent medicine to study and orienting it to a specific clinical use. A solution to these problems is urgently needed.

\section{Problems with the Present Method of Choosing Research Subject and Clinical Orientation}

It is widely acknowledged that the first thing to do in secondary development of an old drug is to define the exact indications of the revised drug. Their difference in applied indications and in varied clinical situations where they were used makes the redevelopment work meaningful. A clear definition of the new drug's intended indications is also considered necessary in efficacy evaluation and when selecting from various formulas.

Problems with choosing research subject and clinical orientation in the secondary development of famous and excellent Chinese patent medicines are summarized as follows:

1. Selection of research topic not well guided by TCM theory;

2. Evidence to support the topic selection less than convincing;

3. Inadequate grasp of previous baseline information and indefinite clinical orientation;

4. Lack of scientificity and risk analysis for the restudy plan.

Consequently, the redevelopment of prestigious old patents was a meaningless pile of repeated work with barely encouraging results. The impact on the domestic drug market is that few individual drugs sell over 1 billion RMB whereas about a hundred drug products sell more than 100 million RMB.

\section{A Proposed New Method with Four Steps}

This new method of choosing research topic and orienting the drug to a specific clinical use features a borrowing of concepts from evidence-based medicine. In the field of new drug development, the basic principles of evidence-based medicine remain unchanged but the methodology needs adjustment for ease of application. The scientific expression of the clinical efficacy of Chinese medicine can only be achieved by learning from updated research results and methodology

${ }^{*}$ Corresponding author: Shang Hongcai, Tianjin University of traditional Chinese medicine, Tianjin, China, 300193; E-mail: shanghongcai@foxmail.com

Received December 07, 2012; Accepted December 07, 2012; Published December 10, 2012

Citation: Hongcai S (2013) The Method of Research Subject Chosen and Clinical Orientation in the Secondary Development of Famous and Excellent Chinese Patent Medicines. Altern Integ Med 1:e102. doi: 10.4172/2327-5162.1000e102

Copyright: @ 2013 Hongcai S. This is an open-access article distributed unde the terms of the Creative Commons Attribution License, which permits unrestricted use, distribution, and reproduction in any medium, provided the original author and source are credited. 
of Chinese medicine [2,3]. To be specific, the following four steps will make a good solution.

\section{Gathering the baseline information of target diseases and related Chinese patent medicines}

Evidence-based medicine with its new concepts and methods has brought a fresh air to the secondary development of Chinese patent medicines. With an evidence-based view, researchers need to make a comprehensive search for published literatures about target diseases and related Chinese patent medicine and obtain grey literatures from pharmaceutical companies as references for secondary study. After reviewing literatures, we collect the baseline information on the disease status of patients treated with this drug and learn about the evolution of the therapeutics of related diseases. According to the above information, we can now conclude what role the drug plays in the intervention in a specific disease and gain the right direction for the redevelopment work.

\section{Selecting an initial research topic and defining the medicinal uses and indications of the medicine in question}

A shortcoming of the Chinese patent medicines on the market is that they are hardly distinguishable from each other. Most patent formulas vary only slightly in the proportions of ingredients and have roughly the same effects, the result of poor market management. More precise clinical orientation of these drugs can change this situation and reflect their different characteristics.

This means every patent medicine needs a clear definition of clinical uses and applied indication of its own, which distinguishes it from other similar medicines. A summarization and analysis of results of basic research on the patent formula and its principal ingredients form the basis of the work in this step.

\section{Analyzing the scientificity and risk of the study}

The evolution of global science has posed fresh and tough challenges to traditional Chinese medicine. Now the public demands more than feeling and witnessing its clinical efficacy. They expect researchers to provide objective and compelling evidence and to explain the material background and action mechanism in plain scientific terms. To add to its vague nature of potency, the use of some patent medicines which were supposed to be safe runs the risk of causing health hazards. This is because changes in pharmacy techniques and dosage forms may alter the toxicity of the medicine. Also, some new undesired effects manifest themselves after long years of applying the medicine in clinical practice. For this reason, scientific feasibility evaluation and risk analysis are obligatory in the secondary development of Chinese patent medicines, which used to be neglected.

Scientificity analysis refers to an objective analysis of the results of modern pharmacological studies on Chinese patent medicine and its principal herbal ingredients. On this basis, risk analysis assesses the possibility of causing dangers using the medicine, on the evidence of reports on drug toxicity and adverse effects. Other risks include the possibility that the study cannot be completed or no satisfactory outcome can be reached. All these risk factors should be kept under control.

It is therefore recommended that a panel of experts specializing in pharmacy, toxicology, pharmacology, methodology and clinical medicine be constituted to give a critical analysis of the scientific feasibility of and risks involved in the secondary development of a certain Chinese patent medicine. And this will provide scientific evidence for research protocol design and minimize the risks of clinical experiments.

\section{Making a strategic decision for the research}

Following the above three steps, it is time for enterprise executives, basing their judgment on the results of earlier mentioned analysis, to decide whether to invest in the research project.

\section{A Case Study Using the New Method}

Qishen Yiqi dripping pill is the product of successful secondary development of famous and excellent Chinese patent medicine. We believe that by knowing how the topic was selected in this research and how to define exactly when and for what the drug is best used, could make the work much easier in forthcoming similar studies. The following is a detailed explanation of the four steps in the case of Qishen Yiqi dripping pills.

\section{Selecting a prestigious patent medicine for secondary development}

Evidence-based medicine emphasizes the importance of secondary study of all the best evidence that were collected, rated and categorized. Here, secondary study, also called systematic review, means more than a review article. It is a part of the research process. Among the wide varieties of Chinese patent medicines on the domestic market, few have been reevaluated after being put on the market. In fact, it is from the reevaluation of post-marketing drugs that new perspectives for secondary development could be gained. In this case, we first collected and reviewed all the 53 clinical studies on the secondary prevention of myocardial infarction, and found western drugs have limitations where traditional Chinese medicine can help. Then we conducted a review of literatures on qi-tonifying and blood-invigorating patent medicines for myocardial infarction prevention [4]. After weighing these drugs' limitations against their advantages, we decided to choose Qishen Yiqi dripping pills as a representative of its kind for secondary development.

\section{Orienting Qishen Yiqi dripping pills to a specific clinical use}

We learned from literature analysis that Qishen Yiqi dripping pills can alleviate angina symptoms, prevent left ventricle remodeling and improve the long-term prognosis of coronary heart diseases. Admittedly, the low methodological quality of included studies could render the evidence provided less reliable. Moreover, drug efficacy could be only partially achieved if medicinal uses and indications were inadequately defined. Considering these factors, we defined Qishen Yiqi dripping pills as best used for patients recovering from myocardial infarction. It also echoes the TCM theory of "chai hou fang fu", namely, taking efforts to prevent the reoccurrence of the disease.

\section{Assessing the scientificity and risk of the clinical research}

We made a comprehensive review of modern pharmacological experiments on Qishen Yiqi dripping pills and its principal components (Huangqi, Danshen, Sanqi and Jiangxiang). There is scientific evidence showing that the patent medicine and its ingredients can intervene in various aspects of the pathological process leading to myocardial infarction. For instance, they can stabilize vulnerable plaque and prevent thrombosis by regulating blood lipid and prohibiting platelets from clotting. And they also help to protect damaged heart muscle, prevent ventricle restructuring and improve heart function.

With regard to the risks involved in the research, previous 
toxicologic studies revealed that Qishen Yiqi dripping pills and its major ingredients have neither obvious side effects nor mutagenic and teratogenic properties. There was also no report of obvious adverse effects coming out form stage II-III clinical observations and postmarketing studies. Furthermore, the risk of failure of implementing the research was kept lowest with rigorous design and quality control. Then the rest of the work is left for the researchers to accept the final results, positive or negative. In either case you will have something to learn from.

\section{Weighing benefits against risks and make a strategic decision}

The decision on whether to undertake the study or not should be reached after we have a full understanding and make a critical evaluation of all the existing evidences. Then, we conduct the research if there is sufficient or necessary evidence supportive of the expected results, and will verify the preliminary results in large-sample highquality clinical trials. But when the risks outweigh the benefits, which means there are more evidence against our assumptions, we will have to change our topic or wait for more evidence.

In the case of Qishen Yiqi dripping pills, the research project was successfully launched as there was sufficient evidence in support of its effects on secondary prevention of myocardial infarction. We believe more good evidence will appear, the procedure of evaluating the efficacy of TCM for myocardial infarction prevention will be established and the methodologies of evidence-based medicine will be set up. These will pave the way for subsequent researches.

\section{Conclusions}

Secondary development of prestigious Chinese patent medicine is meaningful work. One reason is that more often than not some of the drugs' pharmacological actions won't be discovered until years after prescribing them in clinical practice. Yet another reason is secondary study, in comparison with original study, spends less time and money while keeping the risk low.

To sum up, this article deals with a new method of choosing research subject and clinical orientation in the secondary development of famous and excellent Chinese patent medicine. The four steps of the method were illustrated in the case of Qishen Yiqi dripping pills, which include selecting the drug for secondary development, orienting it to a specific clinical use, assessing the scientificity and risk of the research and finally making a strategic decision. It is believed that this method helps to achieve optimal risk-benefit ratio and highlights more efficient use of medical resources. Therefore, we strongly recommend it to all the researchers in this field.

\section{References}

1. Xiaohui W (2004) The method of prescription optimization during the secondary development of Chinese patent drugs and some reflections. Master dissertation of Tianjin University of Traditional Chinese Medicine.

2. Shang HC, Li YP, Chen J, Zhang JH, Zhang BL (2008) New trends for clinical research of traditional Chinese medicine in China. Chin Med J (Engl) 121: 10501051.

3. Shang HC, Zhang BL, Li YP (2008) Clinical orientation of post-marketing Chinese patent drugs: an evidence-based practice. Zhong Xi Yi Jie He Xue Bao 6: 887-890.

4. Junhua Z, Hongcai S, Boli Z (2008) Yiqi Huoxue preparations for the treatment of myocardial infarction: a systematic review. China Journal of Traditional Chinese Medicine and Pharmacy 23: 300-306. 